\title{
Local capillary supply in muscle is not determined by local oxidative capacity
}

\author{
Alessandra Bosutti ${ }^{1,2}$, Stuart Egginton ${ }^{3}$, Yoann Barnouin ${ }^{2}$, Bergita Ganse ${ }^{4, *}$, Jörn Rittweger ${ }^{4}$ and \\ Hans Degens ${ }^{2,5, \neq}$
}

\begin{abstract}
It is thought that the prime determinant of global muscle capillary density is the mean oxidative capacity. However, feedback control during maturational growth or adaptive remodelling of local muscle capillarisation is likely to be more complex than simply matching $\mathrm{O}_{2}$ supply and demand in response to integrated tissue function. We tested the hypothesis that the maximal oxygen consumption $\left(M_{\mathrm{O}_{2} \text {,max }}\right)$ supported by a capillary is relatively constant, and independent of the volume of tissue supplied (capillary domain). We demonstrate that local $M_{\mathrm{O}_{2} \text { max }}$ assessed by succinate dehydrogenase histochemistry: (1) varied more than 100-fold between individual capillaries and (2) was positively correlated to capillary domain area in both human vastus lateralis $(R=0.750$, $P<0.001)$ and soleus $(R=0.697, P<0.001)$ muscles. This suggests that, in contrast to common assumptions, capillarisation is not primarily dictated by local oxidative capacity, but rather by factors such as fibre size, or consequences of differences in fibre size such as substrate delivery and metabolite removal.
\end{abstract}

KEY WORDS: Succinate dehydrogenase, Capillarisation, Muscle, Capillary domains

\section{INTRODUCTION}

Highly oxidative muscles have a denser capillary network than those with a high glycolytic capacity, and within a given muscle, such as rat plantaris, fibres in the oxidative region have a higher capillary density than those in the more glycolytic region (Wüst et al., 2009). This correlation between anatomical capillary supply and tissue oxidative capacity also seems to apply at a smaller scale of biological control, where the local capillary supply to an individual fibre appears to be positively related to its oxidative capacity (Bekedam et al., 2003). Importantly, in such studies, fibre size was not considered and hence the influence of local diffusion distances on cellular oxygenation cannot be assessed. We have previously demonstrated that the local capillary supply to a fibre correlates with its cross-sectional area and is only slightly modulated by its oxidative capacity, but not by phenotype (Egginton and Gaffney, 2010; Wüst et al., 2009). However, if the principle of symmorphosis, which states that structures are matched to functional demand, is valid, then local

\footnotetext{
${ }^{1}$ Department of Medical, Surgery and Health Sciences, University of Trieste, 34100 Trieste, Italy. ${ }^{2}$ School of Healthcare Science, Manchester Metropolitan University, Manchester M1 5GD, UK. ${ }^{3}$ School of Biomedical Sciences, University of Leeds, Leeds LS2 9JT, UK. ${ }^{4}$ Institute of Aerospace Medicine, German Aerospace Centre, Linder Höhe, Cologne 51147, Germany. ${ }^{5}$ Lithuanian Sports University, Kaunas LT-44221, Lithuania.

*Present address: RWTH Aachen University Hospital, Department of Orthopaedic Trauma Surgery, Germany.

${ }^{\ddagger}$ Author for correspondence (h.degens@mmu.ac.uk)
}

Received 10 June 2015; Accepted 4 September 2015 capillarisation in a muscle should be arranged so that maximal oxygen demand per capillary is tightly regulated. To explore whether local feedback results in each capillary serving a similar maximal demand for oxygen, we estimated the supply areas (domains) of individual capillaries (Al-Shammari et al., 2014). Capillary domains provide a good estimate of the tissue oxygenation capacity of a capillary, even in muscles containing a mixture of fibres with different metabolic demand (Al-Shammari et al., 2014), whereas the total volume of mitochondria, as reflected by succinate dehydrogenase activity, in a domain is a reflection of the maximal oxygen demand served by that capillary. We hypothesised that if the primary determinant of local capillary supply was local oxygen demand, then the maximal oxygen demand $\left(M_{\mathrm{O}_{2}, \max }\right)$ supported by each capillary should be similar for each capillary.

\section{MATERIALS AND METHODS}

\section{Human muscle biopsy}

Muscle biopsies were aseptically taken with a Rongeur forceps (Zepf Medizintechnik, Germany) under local anaesthesia ( $2 \mathrm{ml}$ of $1 \%$ lidocaine) from the vastus lateralis (VL) and soleus of young men $(N=10 ; 23-43$ years old), following local ethical approval by the independent ethics committee Ärztekammer Nordrhein, Düsseldorf, Germany (No. 2010426) and written informed consent. The biopsies were taken as part of a bed rest study (ClinicalTrials.gov registration number NCT01655979) using baseline samples only. To facilitate longitudinal orientation, samples were embedded in a silicone tube filled with Optimal Cutting Temperature compound (Scigen ${ }^{\circledR}$ Gardena), frozen in liquid nitrogen and stored at $-80^{\circ} \mathrm{C}$ until analysis. All participants underwent an extensive health screening.

\section{Morphometry}

Serial frozen sections $(8 \mu \mathrm{m})$ were co-stained with biotinylated lectin (Ulex europaeus agglutinin I, Vector Laboratories, UK; $1 \mathrm{~h}, 50 \mu \mathrm{ml}^{-1}$ in $1 \%$ BSA HEPES) and anti-mouse myosin type I (1:100; Novocastra, Leica Biosystems, UK; product code: NCL-MHCs) to reveal capillary locations and Type I fibres, respectively. Sections were subsequently incubated with a secondary goat anti-mouse horseradish peroxidase labelled antibody (30 min, 1:200; Dako, UK) and stained (Vector VIP HRP substrate kit), as described by the manufacturer. The sections were mounted in glycerolgelatine and stored at $4{ }^{\circ} \mathrm{C}$. Serial sections were stained for succinate dehydrogenase (SDH) activity as described previously (Wüst et al., 2009). Briefly, sections were incubated in the dark $\left(20 \mathrm{~min}, 37^{\circ} \mathrm{C}\right.$ in $37 \mathrm{mmol} 1^{-1}$ phosphate buffer, $74 \mathrm{mmol} \mathrm{l}^{-1}$ sodium acetate and $0.4 \mathrm{mmoll}^{-1}$ tetranitroblue tetrazolium, $\mathrm{pH}$ 7.6) (Fig. 1A,B). The optical density at $660 \mathrm{~nm}\left(\mathrm{OD}_{660}\right)$ was determined (Image J; National Institutes of Health, Bethesda, USA) as an index of the aerobic capacity/mitochondrial content of muscle fibres because $\mathrm{OD}_{660}$ is linearly related to fibre $M_{\mathrm{O}_{2} \text {,max }}$ (Wüst et al., 2009). For each image, a separate calibration curve was constructed with a series of filters with a known $\mathrm{OD}_{660}$ to remove potential optical bias related to differences in background intensity and lighting between sections.

Coordinates of fibre outlines and capillary centres (Fig. 1C) were collected using a digitising tablet (MMII 1201, Summagraphics Digitizers, Austin, TX, USA) and analysed (AnaTis, BaLoH Software, http://www. 


\begin{tabular}{|ll|}
\hline \multicolumn{2}{|l|}{ List of abbreviations } \\
$A_{\text {ovl }}$ & area of the overlap of the domain with an individual fibre \\
CAF & capillaries around a fibre \\
DAF & domains supplying a fibre \\
FCSA & fibre cross-sectional area \\
LCFR & local capillary to fibre ratio \\
$M_{\mathrm{O}_{2}, \max }$ & maximal oxygen consumption \\
$P_{\mathrm{O}_{2}}$ & partial pressure of oxygen \\
$\mathrm{SDH}$ & succinate dehydrogenase \\
$\mathrm{VL}$ & vastus lateralis \\
\hline
\end{tabular}

baloh.nl) to calculate capillary domains (Fig. 1C) and parameters related to muscle fibre size (Wüst et al., 2009). A capillary domain was defined as the area of tissue closer to a given capillary than neighbouring capillaries, which is a good estimate of capillary oxygen supply areas in muscles with heterogeneous fibre composition (Al-Shammari et al., 2014). Capillary domains overlap with portions of different fibres surrounding the vessel, and their combined maximal oxygen demand $\left(M_{\mathrm{O}_{2} \text {,max }}\right)$, obtained from the same fibres in a serial section stained for $\mathrm{SDH}$, was calculated as:

$$
M_{\mathrm{O}_{2}, \max }=\sum\left(\mathrm{OD}_{\mathrm{SDH}} \times A_{\mathrm{ovl}}\right),
$$

where $\mathrm{OD}_{\mathrm{SDH}}$ is the $\mathrm{OD}$ of $\mathrm{SDH}$ for an overlap (domain) area, and $A_{\mathrm{ovl}}$ is the area of the overlap of the domain with an individual fibre (Fig. 1D-F). The $M_{\mathrm{O}_{2} \text {,max }}$ was calculated for an average of 160 capillary domains per muscle and individual. Local capillary to fibre ratio (LCFR) is the sum of domain fractions overlapping a given fibre (Fig. 1D-F) and thus is an index of the capillary supply to that fibre and considers the influence of contiguous capillary supply areas in terms of 'supply equivalents' at maximum perfusion/consumption. The number of domains overlapping a fibre (DAF) provides an index of any capillary supplying that fibre, and DAF and LCFR thus give complementary information. Using the assumption that $1 \mathrm{~mol}$ of oxygen is 22.4 litres and the density of muscle is $1 \mathrm{~kg} \mathrm{l}^{-1}$, the $M_{\mathrm{O}_{2} \text {,max }}$ in a

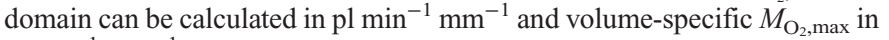
$\mathrm{ml} \mathrm{kg}{ }^{-1} \mathrm{~min}^{-1}$ (Bekedam et al., 2003).

\section{Statistics}

Step-wise regression was performed to assess the impact of fibre type, size and mass-specific $M_{\mathrm{O}_{2} \text {, max }}$ on LCFR and DAF. The correlations between $M_{\mathrm{O}_{2} \text {,max }}$ and domain area were determined by Spearman correlation coefficients because Shapiro-Wilk tests indicated that the data were not normally distributed.

\section{RESULTS AND DISCUSSION}

We confirm that the LCFR correlated positively with fibre crosssectional area (FCSA) in both human VL (Fig. 2A; $R=0.576$ and $R=0.625$ when also including mass-specific $M_{\mathrm{O}_{2}, \max }$ in the model; both $P<0.001$ ) and soleus muscle (Fig. $2 \mathrm{~B} ; R=0.578$ and $R=0.591$, respectively; $P<0.001)$. LCFR per fibre perimeter, a measure of the capillary-fibre contact area, was positively related to the massspecific $M_{\mathrm{O}_{2}, \text { max }}$ in both VL (Fig. 2C; $R=0.329 ; P<0.001$ ) and soleus (Fig. 2D; $R=0.138 ; P=0.002$ ) muscles. Step-wise linear regression revealed that the number of DAF - a functionally more realistic index than 'capillaries around a fibre' - was primarily determined by FCSA; correlations improved when mass-specific $M_{\mathrm{O}_{2} \text {, max }}$ was also included in the model (VL: $R=0.470$ versus 0.508 ; both $P<0.001$; soleus: $R=0.497$ versus 0.510 ; both $P<0.001)$. Only in the soleus did inclusion of fibre type improve the correlation further $(R=0.521 ; P=0.043)$. Intriguingly, in both VL (Fig. 2E) and soleus (Fig. 2F), the $M_{\mathrm{O}_{2} \text {,max }}$ per capillary varied
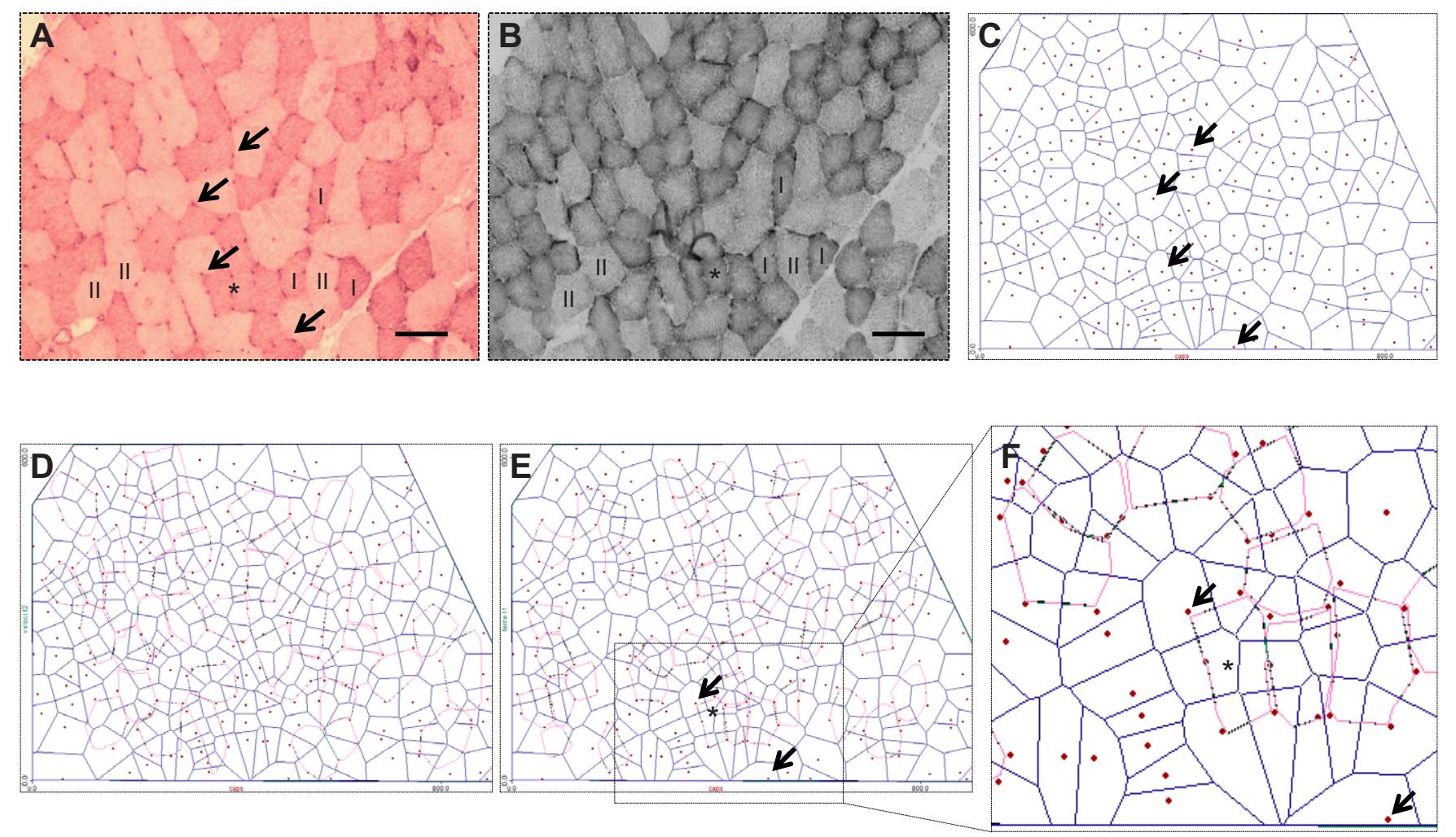

Fig. 1. The relationship between muscle capillary supply area and aerobic capacity. Frozen sections of human vastus lateralis muscle biopsies stained with (A) lectin (Ulex europaeus) to localise capillaries and (B) succinate dehydrogenase as an index of mitochondrial activity. (C) Capillary domains represent the area of tissue closer to a given capillary (red dots) than neighbouring capillaries. (D,E) Overlap of domains with fast and slow fibres, respectively. (F) Magnified region to illustrate overlap of domains (blue outlines) and slow fibres (pink outlines). I: type I fibres; II: type II fibres; arrows indicate corresponding capillary locations in each panel; asterisks identify the same fibre in each panel. Scale bars: $100 \mu \mathrm{m}$. 

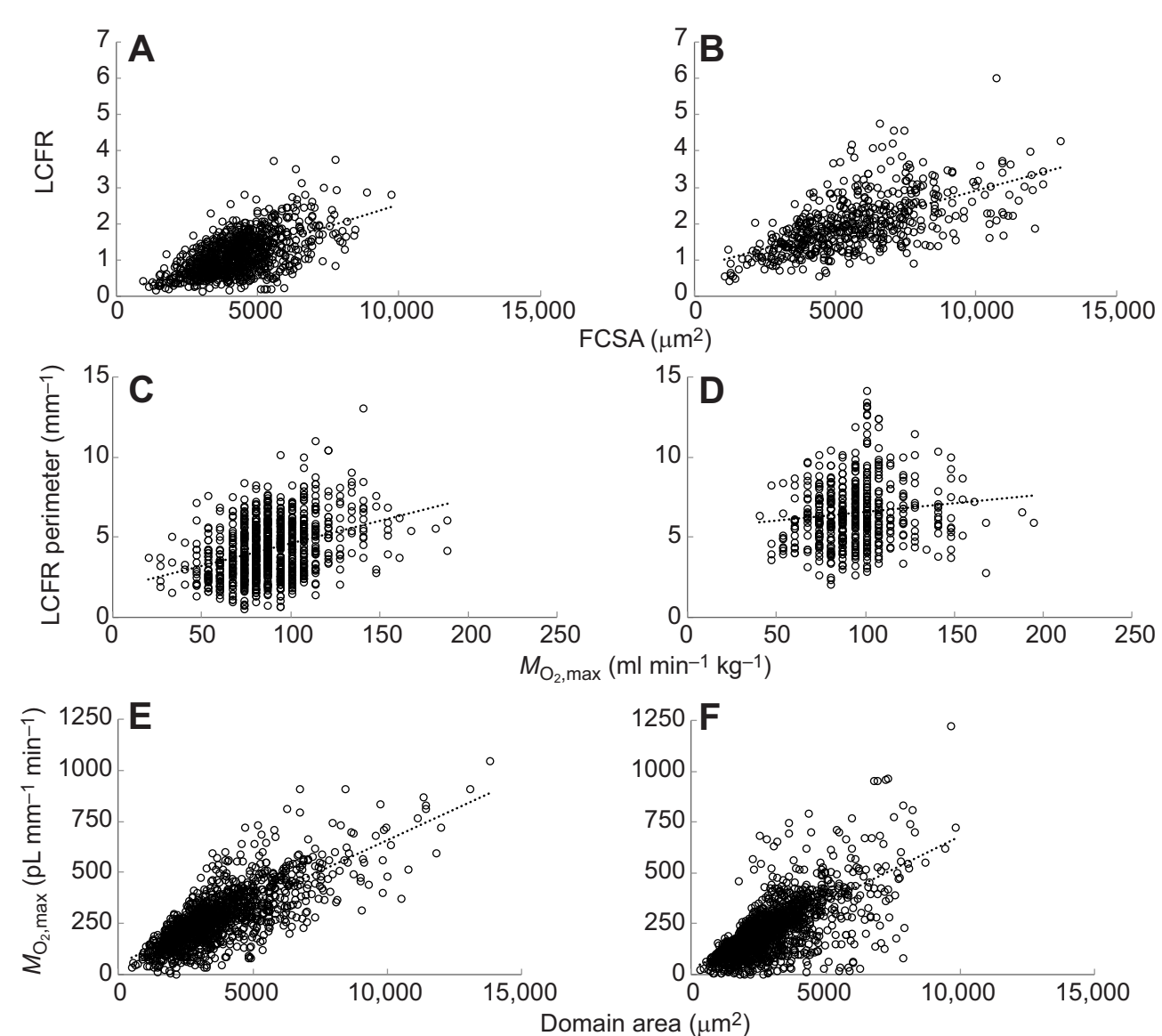

Fig. 2. Relationship between local maximal oxygen demand for individual capillaries and their respective domain area. There is a positive correlation between local capillary to fibre ratio (LCFR) and fibre cross-sectional area (FCSA) in (A) human vastus lateralis (VL) $(R=0.576$ and $R=0.625$ when also including massspecific $M_{\mathrm{O}_{2}}$, max in the model; both $P<0.001)$ and $(\mathrm{B})$ soleus muscles $(R=0.578$ and $R=0.591$, respectively; $P<0.001)$. The $L C F R$ per fibre perimeter, a measure of the capillary-fibre contact area, is positively related to the estimated mass-specific $M_{\mathrm{O}_{2} \text {, max }}$ of fibres in (C) the $\mathrm{VL}(R=0.329 ; P<0.001)$ and $(\mathrm{D})$ soleus $(R=0.138 ; P=0.002)$ muscle. A positive correlation is also observed between estimated domain $M_{\mathrm{O}_{2} \text {,max }}$ and domain area in (E) VL $(R=0.750, N=1443$ capillaries, $P<0.001, R=0.822 \pm 0.017$ for regression lines from each of the 10 individuals; mean \pm s.e.m.) and $(F)$ soleus $(R=0.697, N=1742, P<0.001, R=0.705 \pm$ $0.038)$ muscle. The VL and soleus contained $36 \pm 4 \%$ and $75 \pm 4 \%$ (means \pm s.e.m.) type I fibres, respectively. from almost 0 to more than $1000 \mathrm{pl} \mathrm{mm}{ }^{-1} \min ^{-1}$. Also, $M_{\mathrm{O}_{2} \text {,max }}$ was positively correlated with domain area in VL (Fig. 2E) and soleus (Fig. 2F). Thus, capillaries with larger oxygen supply areas supply a larger volume of mitochondria, and hence support a potentially larger maximum oxygen flux. These observations may require a fundamental review of ideas about determinants of local muscle capillary supply.

Even at the whole muscle level, a poor correlation with gross capillarisation was found in several species across a 17-fold range in muscle oxidative capacity (Maxwell et al., 1980). Capillary growth can occur without an increase in oxidative capacity, e.g. following selective stimulation of fast fibres (Egginton and Hudlická, 2000). While there may still be temporal coupling, because not all capillaries are perfused at any given moment and perfused vessels may have different flows adapted to the local demand for oxygen, it is unlikely that such perturbations during exercise can account for the more than $1000 \mathrm{pl} \mathrm{mm}{ }^{-1} \mathrm{~min}^{-1}$ range in local $M_{\mathrm{O}_{2} \text {,max }}$ among capillaries, as even at rest, all capillaries will have been perfused in as little as $20 \mathrm{~s}$ (Hargreaves et al., 1990). Another possibility is that the positive relationship between local $M_{\mathrm{O}_{2} \text {,max }}$ and capillary domain size reflects a compensation for a reduced oxygen diffusion gradient due to the decreasing microvascular $P_{\mathrm{O}_{2}}$ from the arteriolar to venular end of capillaries (Egginton and Gaffney, 2010). While these factors may help to match temporal oxygen supply and demand, they do not explain why local structural capillary supply correlates poorly with local maximal oxygen demand, a violation of the symmorphosis principle that assumes structures are matched to functional demand. While the heterogeneity of capillary spacing does have an impact on tissue oxygenation (Egginton and Gaffney, 2010), it is apparently not regulated to maintain $M_{\mathrm{O}_{2} \text {,max }}$ per capillary. The capillary-fibre exchange area, however, may be a better reflection of the capacity for oxygen flux, as suggested by the greater correlation between peak oxygen consumption with the capillary-fibre contact area than with capillary density (Hepple et al., 1997). Lack of significant correlations between fibre oxidative capacity and the local capillary density in both rat and human muscle (Wüst et al., 2009), but significant correlations between LCFR or DAF per fibre perimeter and fibre oxidative capacity, support this suggestion. Finally, the high volume of mitochondria in larger domains may serve to enhance flux of oxygen by exerting an extraction pressure, hence increasing total respiration rate, even if individual mitochondria are working submaximally under conditions of reduced oxygen tension. It is striking, however, that the local capillary supply was more tightly related to FCSA than fibre oxidative capacity. Consistent with these observations, fibre hypertrophy and angiogenesis during muscle overload have a similar time course (Plyley et al., 1998), further supporting a coupling between fibre size and local muscle capillarisation. Such a coupling is, in part, explicable by the fact that not only muscle fibres and satellite cells, but also endothelial cells act as mechanotransducers and may secrete factors with reciprocal effects in response to mechanical deformation (Christov et al., 2007).

Whatever the cause of this novel observation, the data indicate that: (1) maximal oxygen demand supported by individual capillaries varies enormously (more than $1000 \mathrm{pl} \mathrm{mm}^{-1} \mathrm{~min}^{-1}$ ) and (2) muscle fibre size rather than local maximal oxygen demand is a prime determinant of local muscle capillarisation.

\section{Acknowledgements}

We thank all the participants for providing muscle biopsies.

Competing interests

The authors declare no competing or financial interests. 


\section{Author contributions}

A.B. performed the experiments, analysis and data interpretation. S.E. and H.D. interpreted the data and wrote the manuscript. H.D. designed the experiments, helped with the analysis and data interpretation, and wrote the first draft of the manuscript. J.R. and B.G. took the muscle biopsies. All authors discussed the results and approved the final manuscript.

\section{Funding}

We are grateful for support from the European Space Agency (AO-06-BR).

\section{References}

Al-Shammari, A. A., Gaffney, E. A. and Egginton, S. (2014). Modelling capillary oxygen supply capacity in mixed muscles: capillary domains revisited. J. Theor. Biol. 356, 47-61.

Bekedam, M. A., van Beek-Harmsen, B. J., Boonstra, A., van Mechelen, W., Visser, F. C. and van der Laarse, W. J. (2003). Maximum rate of oxygen consumption related to succinate dehydrogenase activity in skeletal muscle fibres of chronic heart failure patients and controls. Clin. Physiol. Funct. Imaging 23, 337-343.

Christov, C., Chretien, F., Abou-Khalil, R., Bassez, G., Vallet, G., Authier, F.-J., Bassaglia, Y., Shinin, V., Tajbakhsh, S., Chazaud, B. et al. (2007). Muscle satellite cells and endothelial cells: close neighbors and privileged partners. Mol. Biol. Cell 18, 1397-1409.

Egginton, S. and Gaffney, E. A. (2010). Tissue capillary supply - it's quality not quantity that counts! Exp. Physiol. 95, 971-979.

Egginton, S. and Hudlická, O. (2000). Selective long-term electrical stimulation of fast glycolytic fibres increases capillary supply but not oxidative enzyme activity in rat skeletal muscles. Exp. Physiol. 85, 567-573.

Hargreaves, D., Egginton, S. and Hudlická, O. (1990). Changes in capillary perfusion induced by different patterns of activity in rat skeletal muscle. Microvasc. Res. 40, 14-28.

Hepple, R. T., Mackinnon, S. L., Goodman, J. M., Thomas, S. G. and Plyley, M. J. (1997). Resistance and aerobic training in older men: effects on VO2peak and the capillary supply to skeletal muscle. J. Appl. Physiol. 82, 1305-1310.

Maxwell, L. C., White, T. P. and Faulkner, J. A. (1980). Oxidative capacity, blood flow, and capillarity of skeletal muscles. J. Appl. Physiol. Respir. Environ. Exerc. Physiol. 49, 627-633.

Plyley, M. J., Olmstead, B. J. and Noble, E. G. (1998). Time course of changes in capillarization in hypertrophied rat plantaris muscle. J. Appl. Physiol. 84, 902-907.

Wüst, R. C. I., Gibbings, S. L. and Degens, H. (2009). Fiber capillary supply related to fiber size and oxidative capacity in human and rat skeletal muscle. Adv. Exp. Med. Biol. 645, 75-80. 